\title{
Predicted Area Under the First Moment Curve Percent Extrapolation
}

National Cancer Institute

\section{Source}

National Cancer Institute. Predicted Area Under the First Moment Curve Percent

Extrapolation. NCl Thesaurus. Code C85790.

The percent of the predicted area under the first moment curve (AUMC) that is extrapolated to infinity. 